\title{
Coupling of near-grazing microwave photons to surface plasmon polaritons via a dielectric grating
}

\author{
A. P. Hibbins and J. R. Sambles \\ Thin Film Photonics Group, School of Physics, University of Exeter, Exeter EX4 4QL, United Kingdom \\ C. R. Lawrence \\ Defence Evaluation and Research Agency, Farnborough GU14 OLX, United Kingdom
}

(Received 8 November 1999)

\begin{abstract}
A dielectric grating on top of a planar metal substrate is shown to couple near-grazing microwave photons to surface plasmon polaritons (SPPs). It is shown that when the grating grooves are oriented such that they are parallel to the plane of incidence $\left(\varphi=90^{\circ}\right)$, coupling to SPPs with both $s$ - and $p$-polarized photons is possible at three different energies. It is demonstrated that one mode is coupled via $p$-polarized radiation and the other two modes are both coupled via $s$-polarized radiation. A multilayer, multishape differential grating theory allows the identities of each of the modes to be confirmed by modeling the electromagnetic fields above the metal substrate. In addition, a comparison between the experimentally derived reflectivity scans and the theoretical model is made.
\end{abstract}

PACS number(s): 42.79.Dj, 52.25.Sw, 52.70.Gw, 42.25.Fx

\section{INTRODUCTION}

The electromagnetic response of periodically corrugated surfaces has been the subject of scientific study over the past one hundred years. In particular, the series of "anomalies", [1], which take the form of sharp changes in the reflected intensity of $p$-polarized (transverse magnetic, TM) radiation from a metallic grating when the direction of the grooves is parallel to the plane of incidence, have stimulated much interest. They are associated with the now well-understood surface plasmon polariton (SPP) and comprise a coupling of the incident electromagnetic field to the oscillation of the surface charge density at a metal-dielectric interface. The SPP is strongly confined at the interface and this results in the dispersion of the mode being extremely sensitive to the boundary along which it propagates. This sensitivity may be exploited through suitable reflectivity measurements to determine the profile of the grating or the complex permittivities of the media surrounding it [2].

The SPP that propagates along a planar boundary in a single-interface system is characterized by a phase velocity greater than that of the incident light, and hence the mode may neither radiate nor be directly coupled. In order to resonantly excite this mode, the in-plane momentum of the radiation must be suitably enhanced. This may be achieved by using a coupling prism in the Otto [3] or KretschmannRaether [4] geometry, or alternatively surface roughness may be employed. A controlled version of the latter is a sinusoidal diffraction grating where the periodically corrugated interface provides the necessary in-plane wave vector enhancement in integer multiples of the grating wave vector $\mathbf{k}_{g}\left[\mathbf{k}_{g}=\left(2 \pi / \lambda_{g} \hat{\mathbf{x}}\right)\right]$. Here $\lambda_{g}$ is the grating pitch and $\hat{\mathbf{x}}$ is the unit direction vector along the grating surface perpendicular to the direction of the grooves. Hence we may resonantly excite the SPP when its wave vector $k_{\text {SPP }}$ satisfies

$$
\mathbf{k}_{\mathrm{SPP}}=n_{d} \mathbf{k}_{0} \sin \theta \pm N \mathbf{k}_{g}
$$

where $N$ is an integer, $n_{d}=\varepsilon_{d}^{1 / 2}$ is the refractive index of the dielectric, and $\lambda_{0}=2 \pi / k_{0}$ is the vacuum wavelength of the incident radiation.

Many previous workers have investigated the dispersion of the SPP that propagates along a corrugated metaldielectric boundary at visible [5,6], infrared [7], and microwave [8] wavelengths. It has generally been considered possible to couple only incident $s$-polarized (TE) radiation to the SPP when the grating grooves are oriented such that they are parallel to the plane of incidence $\left(\varphi=90^{\circ}\right)$. However, recent work [9] has shown that for sufficiently deep grooves coupling is also possible with incident $p$-polarized (TM) radiation. Here, another mechanism for coupling $p$-polarized radiation to the SPP when the grating grooves are oriented in this way is established. A two-interface geometry is utilized, where the corrugated boundary, which provides the coupling mechanism to the SPP, is separated from the planar one along which the mode propagates. A similar geometry appears to have been first used by Müller et al. [10] who successfully coupled visible $(632.8 \mathrm{~nm})$ radiation to the SPP mode. Their sample consisted of an evaporated silver substrate, overcoated with a $5 \mathrm{~nm}$ layer of gold and a photoresist layer containing an interferographically produced grating. Other recent studies involving dielectric grating samples include that of Salakhutdinov et al. [11]. They provided a theoretical and experimental study of a metal-dielectric grating that shows increased diffraction efficiency over a conventional metallic grating due to the excitation of "leaky" guided modes in the dielectric layer. In addition, Seshadri [12] has theoretically investigated the reflection of radiation from a dielectric grating sample with a metallic substrate as a function of the angle of incidence. On excitation of SPPs and guided modes in the dielectric film, peaks were observed in the calculated absorption spectra, and it was shown that the angular response of the experimentally determined reflection coefficient may be used to obtain estimates of the average thickness of the film and amplitude of the grating. 
The experiments undertaken here are carried out as a function of wavelength and azimuthal angle of incidence $(\varphi)$. Defining $\varphi$ as the angle between the plane of incidence and the $x$ axis, Eq. (1) generalizes to

$$
k_{\mathrm{SPP}}^{2}=\varepsilon_{d}^{\mathrm{eff}} k_{0}^{2} \sin ^{2} \theta+N^{2} k_{g}^{2} \pm 2\left(\varepsilon_{d}^{\mathrm{eff}}\right)^{1 / 2} N k_{g} k_{0} \sin \theta \cos \varphi,
$$

where $\varepsilon_{d}^{\text {eff }}(t)$ is an effective complex permittivity of the dielectric that is dependent on the thickness of the overlayer $\left(\varepsilon_{d}^{\text {eff }} \rightarrow \varepsilon_{d}\right.$ as $\left.t \rightarrow \infty\right)$. Notice that all the surface vectors are collinear when $\varphi=0^{\circ}$.

In previous studies $[8,13]$, it has been shown that one may couple incident microwave radiation into a SPP $\left(\mathrm{TM}_{0}\right.$ mode $)$ propagating along a metal-dielectric interface by using a diffraction grating of suitable pitch. If this grating profile is formed in the metal substrate then the width of the observed resonances will depend only on the radiative decay of the mode and hence the amplitude of the grating [14,15]. This is because no energy may be dissipated into the substrate since metals behave as near-perfect conductors at microwave frequencies. However, it has been shown [13] that it is also possible to couple radiation into the SPP by depositing a corrugated wax overlayer on top of a planar metal substrate. The grating again provides the mechanism for radiative decay, but as the wax is slightly absorbing the SPP may also decay via loss into the wax. In this situation, both damping terms will contribute to the width of the surface plasmon resonance. In addition, if the grating grooves are neither parallel nor perpendicular to the plane of incidence $(\varphi$ $\left.\neq 0^{\circ}, 90^{\circ}\right)$, then the polarization of the reradiated signal will be different from that of the incident beam and a polarization conversion [16] signal may be detected. If one chooses to detect linearly polarized radiation, then this mechanism provides a third channel into which energy may be transferred from the detected beam-it is "absorbed" by the polarization setting of the detector.

In this study, the reflectivity of near-grazing microwave radiation is recorded as a function of wavelength and azimuthal angle of incidence from a sample consisting of a slightly absorbing, thin dielectric (wax) grating on top of a near-perfectly-conducting metallic (aluminum-alloy) substrate. Such a sample allows the coupling of both p- (TM) and $s$ - (TE) polarized incident photons to the SPP at all orientations other than $\varphi=0^{\circ}$, and according to the condition stated in Eq. (2). The grating pitch and wavelength range are chosen so that at $\varphi=90^{\circ}$ the grating is effectively "zero order," i.e., the only order propagating is the specularly reflected beam. Hence any detected losses on excitation of any modes propagating in the system recorded at $\varphi=90^{\circ}$ are solely due to absorption of energy into the dielectric layer (since there is no polarization conversion at this angle). Wavelength-dependent reflectivity scans are fitted to a rigorous grating modeling theory [17] using the corrugation amplitude, wax thickness, polar angle of incidence, and the absorption of the wax $\left(\varepsilon_{d}^{\prime \prime}\right)$ as fitting parameters. As grazing incidence is approached, one observes an evolution of the $s$-coupled SPP into two different modes. Utilization of the modeling theory to predict the field distribution throughout the sample provides an explanation of this phenomenon.

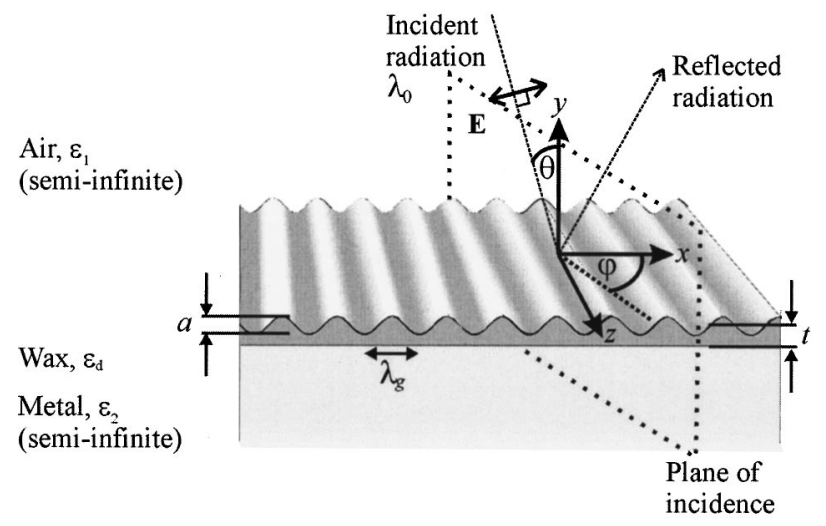

FIG. 1. Schematic diagram illustrating the sample, coordinate system, and experimental geometry used in this study. The direction of the electric field vector $\mathbf{E}$ is illustrated for the situation when $p$-polarized (transverse magnetic, TM) radiation is incident. Here $t$ is the average thickness of the wax overlayer, $\lambda_{0}$ is the wavelength of the incident radiation, $\lambda_{g}$ is the grating pitch, and $a$ is the grating amplitude.

This paper is structured in the following manner. Section II concerns the design and manufacture of the sample, together with a description of the experimental apparatus and method used to record the reflectivity data. Section III contains the experimentally determined reflectivities, and provides a comparison with the predictions from the grating modeling theory. Section IV details the primary result that, when the grating grooves are oriented such that they are parallel to the plane of incidence $\left(\varphi=90^{\circ}\right)$, coupling to SPPs with both $s$ - and $p$-polarized photons is possible at three different energies. Grating theory is used to identify the coupling mechanism for each of the modes by modeling the electromagnetic fields above the metal substrate. Finally, Sec. V provides a brief summary of the work presented.

\section{EXPERIMENTAL PROCEDURE}

It is necessary to design a sample whose wax grating pitch $\left(\lambda_{g}\right)$, amplitude $\left(a_{1}\right)$, and thickness $(t)$ will permit resonant coupling to the SPP using near-grazing photons within the available wavelength range $\left(7.5<\lambda_{0}<11.3 \mathrm{~mm}\right)$. By utilizing the grating modeling theory, the optimum parameters required in order to achieve these results are deduced. The sample chosen has a purely sinusoidal top interface profile, $A(x)=a_{1} \cos 2 \pi x / \lambda_{g}, \quad$ where $a_{1}=1.50 \mathrm{~mm}$ and $\lambda_{g}$ $=15.00 \mathrm{~mm}$, with mean thickness of wax $t=1.60 \mathrm{~mm}$. The real part of the complex permittivity of the wax has been determined via interferographic reflectivity measurements from a planar slab of known thickness, and the imaginary part has been measured by fitting the predictions from the grating modeling theory to reflectivity measurements in a previous study [13].

The sample is prepared by filling a metallic, square tray of side approximately $400 \mathrm{~mm}$ and depth $5 \mathrm{~mm}$ with hot wax and allowing it to cool. A sinusoidal template is manufactured with a pitch of $\lambda_{g}=15.00 \mathrm{~mm}$ and fundamental amplitude of $a_{1}=1.50 \mathrm{~mm}$. It is used to remove unwanted wax from the sample by carefully dragging it across the surface until the required grating profile is obtained (Fig. 1).

Figure 2 illustrates the experimental arrangement used to 


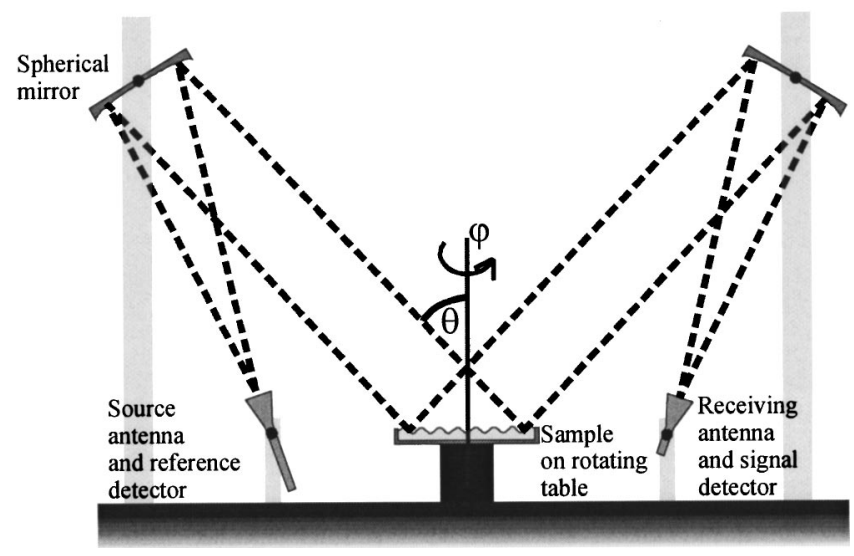

FIG. 2. Schematic diagram illustrating the apparatus used to measure the wavelength- and azimuthal-angle-dependent response from the sample studied in this work.

record the reflectivity from the sample, and Fig. 3 shows a schematic representation of the interconnecting components of the system. The arrangement shown in Fig. 2 has been developed in order to reduce the undesirable effects of spherical wave fronts that may provide excitation of the SPP even without the grating. The problem of near-spherical wave fronts arises from the standard gain horn geometry and the large radiation wavelength. Typically, the source beam divergence is some $20^{\circ}$. By placing the transmitting horn at the focus of a $2 \mathrm{~m}$ focal length mirror, the incident beam is well collimated and the beam divergence may be reduced to approximately $1^{\circ}$. A second mirror is positioned to collect the specularly reflected beam from the grating and focus it into a detector. The aluminum-alloy mirrors have been milled with a diameter of $450 \mathrm{~mm}$ and a radius of curvature $R=4 \mathrm{~m}$.

Variation of the magnitude of the incident wave vector in the plane of the grating may be achieved by scanning either wavelength $\left(\lambda_{0}\right)$ or angle of incidence $(\theta$ or $\varphi)$. In this study the reflectivity data are taken as a function of both wavelength $\left(7.5<\lambda_{0}<11.3 \mathrm{~mm}\right)$ and azimuthal angle of incidence $\left(0<\varphi<90^{\circ}\right)$, fixing the polar angle of incidence at $\theta \approx 80^{\circ}$. Figure 1 illustrates the coordinate system used to describe the geometry of the sample.

The source and receiving horn antennas are set to pass either $p$ - (TM) or $s$ - (TE) polarizations, which enables the measurement of $R_{p p}, R_{p s}, R_{s s}$, and $R_{s p}$ reflectivities, where

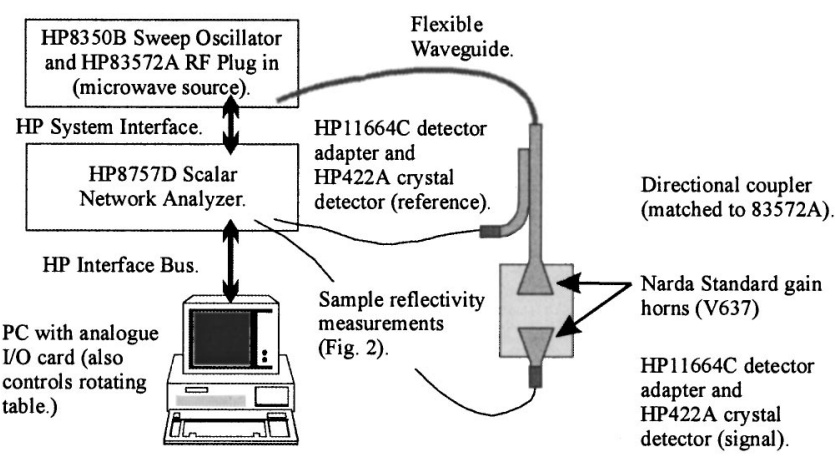

FIG. 3. Schematic representation of the interconnecting components used to record reflectivities in the $7.5-11.3 \mathrm{~mm}$ wavelength range. the subscripts refer to the incident and detected polarizations in that order. To account for any fluctuations in the emitted power from the source, the analyzer divides the output from the signal detector by that from the reference. The normalized reflectivities over the entire frequency range are downloaded from a scalar network analyzer to a PC and are saved to disk. The resulting wavelength-dependent reflectivities from the sample are normalized by comparison with the reflected signal from a flat metal plate of exactly the same dimensions.

In the process of forming the grating profile, wax from approximately $1 \mathrm{~cm}$ wide strips at the edges of the sample was removed in order to be able to adjust the height of the sinusoidal comb and hence the overlayer thickness. Clearly, reflected radiation from these areas of bare metal substrate may cause interference oscillations in the normalized signal. In addition, at the high polar angle of incidence used in this work, the effective incident beam spot size on the grating may be larger than the sample itself, causing unwanted scattering and possible interference from the sample edges. However, by aperturing the two mirrors with microwave absorbing material, it is possible to restrict the size of the incident beam and reduce the magnitude of the interference oscillations. In addition, placing randomly angled reflecting material on the exposed areas of the metal base ensures that only radiation striking the wax surface will reach the collecting mirror and detector. Any effects associated with diffraction from the edges of the sample or the mirrors, or the finite size of the beam, are also largely removed in the normalization process.

\section{RESULTS}

Figure 4 illustrates two polar gray-scale maps of the normalized experimental $R_{s s}$ and $R_{p p}$ signals from the sample. The data have been plotted as a function of frequency and azimuthal angle of incidence, at a fixed polar angle of incidence $\left(\theta \approx 80^{\circ}\right)$. The response of the sample was recorded in the wavelength range $7.5<\lambda_{0}<11.3 \mathrm{~mm} \quad(40.0>f$ $>26.5 \mathrm{GHz}$ where $f$ represents the frequency) and azimuthal angle range $0^{\circ}<\varphi<90^{\circ}$, and then simply reflected about the axes to produce full $360^{\circ}$ plots. Similar dispersion diagrams were plotted in a previous study, and an explanation is provided there [13] of the way in which the modes can be identified and labeled according to the diffracted order with which they are associated.

The band labeled 1 corresponds to the SPP that is coupled via the second-order diffracted beam $(N= \pm 2)$ and is observed as a slight dip in the $R_{p p}$ signal, and a peak in the $R_{s s}$ response. The Rayleigh anomaly that corresponds to this diffracted order occurs at a slightly lower angle of azimuth than the SPP, but is not visible in the experimental data. (The Rayleigh anomaly is the point at which one of the diffracted orders emerges from the grating at grazing angle, resulting in a sudden redistribution of the available energy.) However, the Rayleigh anomaly associated with the first diffracted order is clearly visible in the $R_{p p}$ data as a dramatic change in reflected intensity in the specular order response (indicated by the arrow). The bands labeled 2, 3, and 4 all correspond to the SPP excited by the evanescent fields associated with this diffracted order $(N= \pm 1)$. Notice also the faint circular 


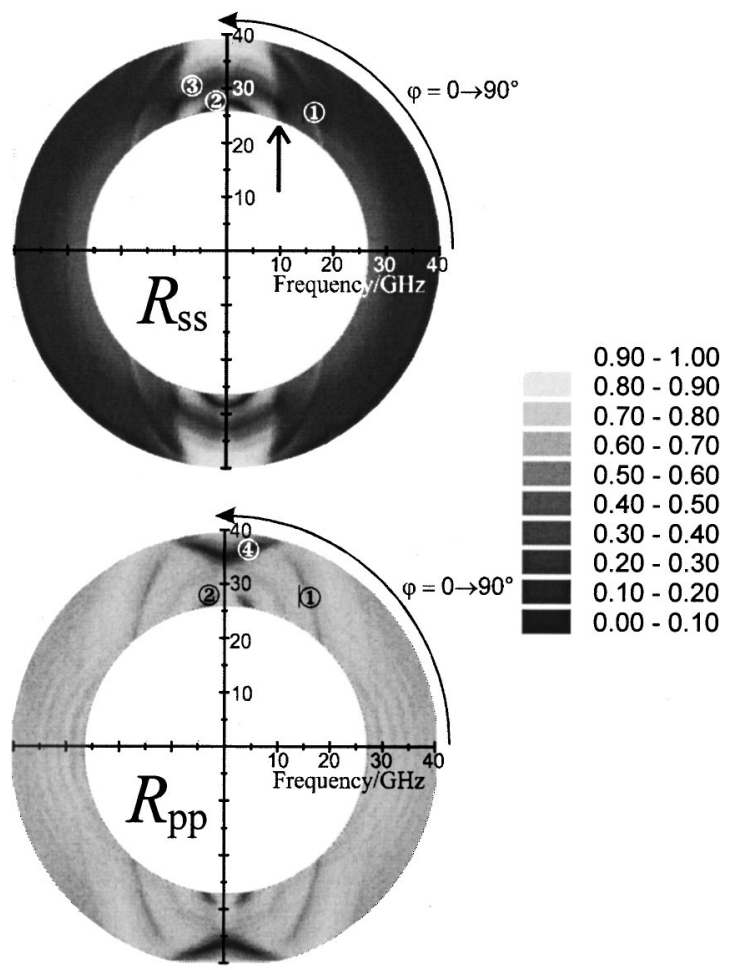

FIG. 4. Normalized $R_{s s}$ and $R_{p p}$ response of the sample as a function of frequency and azimuthal angle of incidence. Data have been recorded between $\varphi=0^{\circ}$ and $\varphi=90^{\circ}$ and reflected about the axes to produce full $360^{\circ}$ polar maps. The band labeled 1 corresponds to the $\pm 2 \mathrm{SPP}$, and the bands labeled 2, 3, and 4 all correspond to the \pm 1 SPP. The arrow on the upper diagram identifies the corresponding \pm 1 Rayleigh anomaly.

rings in the $R_{s s}$ signal around $\varphi=0^{\circ}, 180^{\circ}$ in Fig. 4. These variations, which are weak interference oscillations, are strongest in the data around $\varphi=0^{\circ}, 180^{\circ}$, and hence they are not seen in the slices taken at $\varphi=90^{\circ}$ in Fig. 5 .

Figure 5 illustrates the $R_{s s}$ and $R_{p p}$ response of the sample with the grating orientated so that $\varphi=90^{\circ}$, which is equivalent to taking a slice through the respective plots shown in Fig. 4. It is important to note that, at this azimuthal angle of incidence, the grating wave vector $k_{g}$ is perpendicular to the plane of incidence, and hence no polarization conversion can occur. The reflectivity data recorded at $\varphi=90^{\circ}$ were fitted to the grating modeling theory using the same parameters for both $R_{s s}$ and $R_{p p}$ scans. This theory uses a scattering matrix technique based on a conical version of the differential formalism of Chandezon et al. [18]. It involves the transformation of the mathematics into a coordinate system in which the boundary conditions at the corrugated interface are more readily solved, effectively flattening the surface. This requires that the field amplitudes are represented as a Fourier expansion. The surface itself is also described by a Fourier series:

$$
\begin{aligned}
A(x)= & a_{1} \cos \left(k_{g} x\right)+a_{2} \cos \left(2 k_{g} x\right)+\cdots \\
& +a_{N} \cos \left(N k_{g} x\right)+\cdots
\end{aligned}
$$

where $a_{N}$ provides the first-order scattering mechanism to the $\pm N$ SPP. During the fitting process, the amplitude of the corrugation, thickness of the wax, and polar angle of inci-
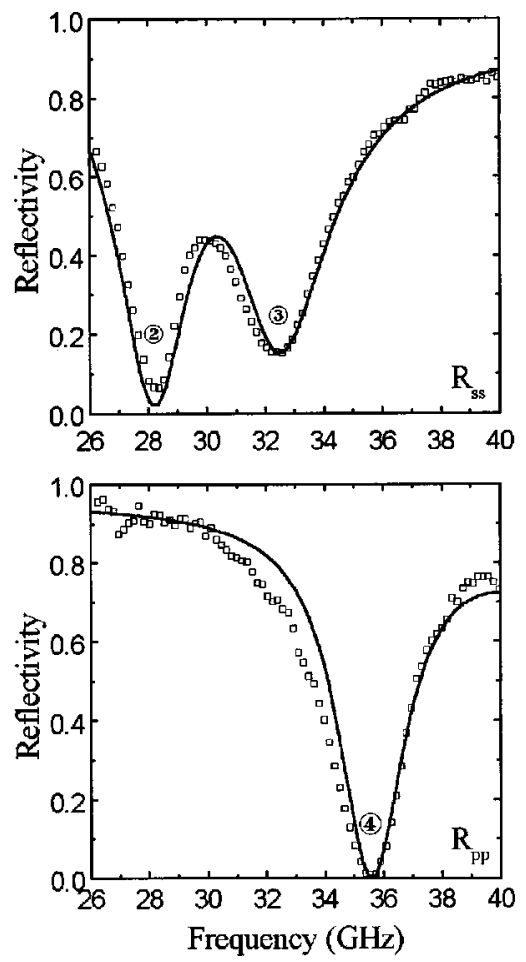

FIG. 5. The normalized $R_{s s}$ and $R_{p p}$ frequency-dependent response $(\square)$ of the sample orientated at $\varphi=90^{\circ}$. The solid lines on each of the graphs correspond to the theoretically modeled results that have been created from the same set of fitting parameters. The two experimental data sets shown have been extracted from the data shown in Fig. 4.

dence are all allowed to vary from their estimated values. The permittivity of the wax is initially assumed to be identical to that determined in the previous study [13] $\left(\varepsilon_{d}\right.$ $=2.29+0.04 i)$; however, the imaginary part is allowed to vary in the fitting procedure. The azimuthal angle is set to $\varphi=90^{\circ}$ and the permittivities of the metal and air are assumed to be $\varepsilon_{2}=-10^{4}+10^{6} i$ and $\varepsilon_{1}=1.0+0.0 i$, respectively. In addition, the pitch of the grating is independently measured to be $\lambda_{g}=15.01 \pm 0.03 \mathrm{~mm}$. Distortion of the grating profile $\left(a_{2}, a_{3}\right)$ is also introduced; however, it does not improve the average quality of the fits, confirming the sinusoidal nature of the corrugation.

The best comparison between the model and the experimentally derived reflectivities is achieved when the following fitting parameters are used: $a_{1}=1.49 \pm 0.01 \mathrm{~mm}, t$ $=1.59 \pm 0.01 \mathrm{~mm}, \theta=80.0 \pm 0.5^{\circ}$, and $\varepsilon_{d}^{\prime \prime}=0.061 \pm 0.004$. Comparisons between the predictions using these parameters and the experimental data are shown in Fig. 5.

\section{DISCUSSION}

The fitted $R_{p p}$ and $R_{s s}$ reflectivity data recorded at $\varphi$ $=90^{\circ}$ and shown in Fig. 5 illustrate good agreement between the theory and the experimental results. Clearly, the known amplitude of the grating template agrees with the amplitude determined via the fitting procedure. In addition, the value of the imaginary part of the dielectric constant of the wax determined here compares favorably to that measured in a previous study [13].

Figure 4 illustrates the dispersion of modes that are sup- 

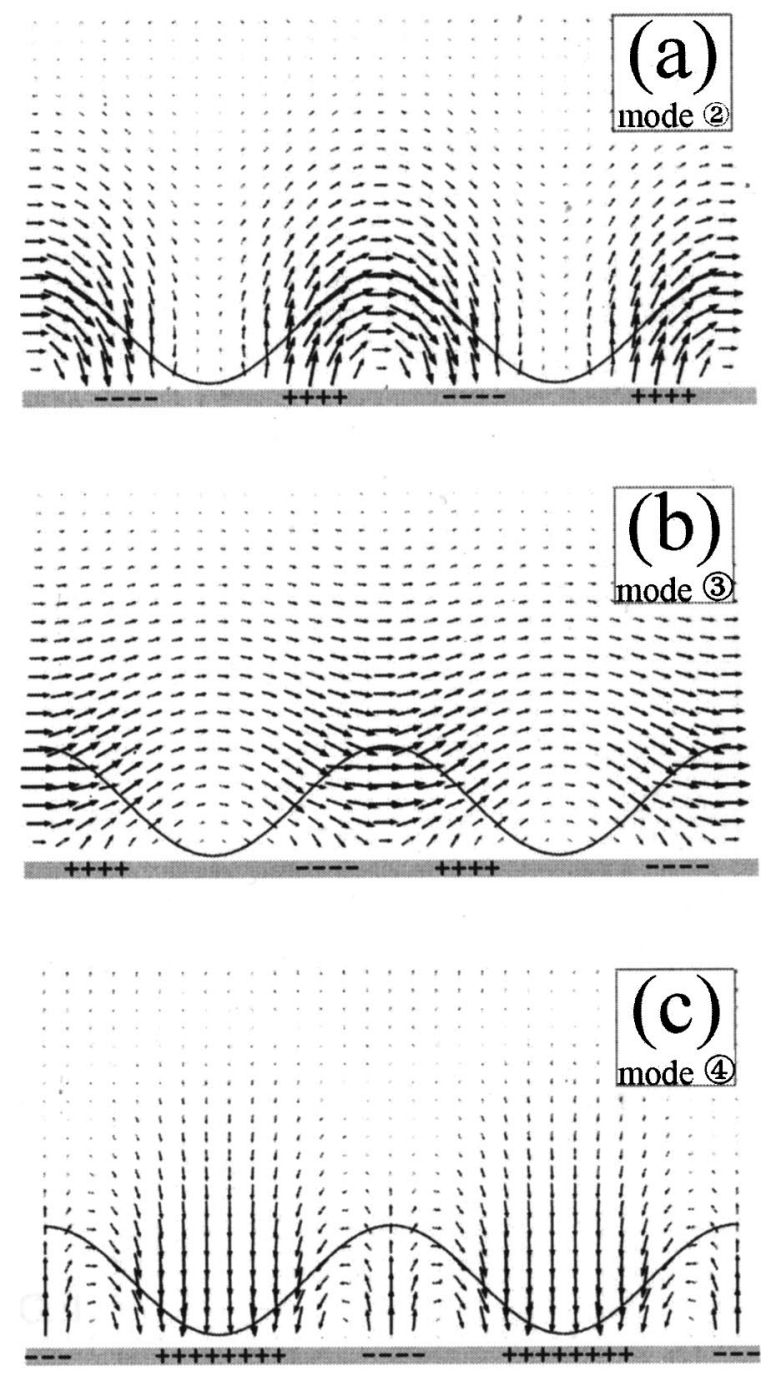

FIG. 6. The predicted $E_{x y}$ vector fields corresponding to each of the three resonances shown in Fig. 5 at the instant in time of strongest coupling. (a) corresponds to the low-frequency $s$-coupled mode 2 ; (b) to the high-energy $s$-coupled mode 3; and (c) to the $p$-coupled mode 4.

ported by the sample and are excited using near-grazing microwave radiation in the $26.5-40 \mathrm{GHz}$ frequency range $\left(11.3>\lambda_{0}>7.5 \mathrm{~mm}\right)$. This section will demonstrate that these modes correspond to the resonance condition of the surface plasmon polariton.

The experimentally measured response of the sample in the special case when the grooves are oriented so that they are parallel to the plane of incidence $\left(\varphi=90^{\circ}\right)$ is shown in Fig. 5. In this situation, polarization conversion is not possible, and since no orders are propagating in the system other than the specular beam, any energy loss in the reflected signal on resonance of the SPP can be lost only into the medium surrounding the interface. It is possible to verify the resonance condition of the SPP by using the grating modeling theory to plot the vector electric fields in the $x y$ plane. A strong local component of the electric field vector normal to the metal-wax interface $\left(E_{y}\right)$ induces a periodic oscillation of surface charge along the boundary, showing the coupling of incident radiation to a surface mode. Figure 6 illustrates the predicted $E_{x y}$ instantaneous vector fields at $\varphi=90^{\circ}$ for the three resonance wavelengths and it is clear in each case that there is a strong normal component. These graphs have been calculated at an instant in time at which the coupling strength to the SPP is strongest (maximum $E_{y}$ ).

It is important to consider how the SPP is created along the metal boundary in each of the resonance conditions described above, remembering that it is only the local normal component of the electric field that excites surface charge and thus couples to a SPP. When $s$-polarized radiation at near-grazing incidence impinges on the sample, there is no component of the electric field vector normal to the metal interface to directly create the required surface charge. However, there is always a component of $\mathbf{E}$ normal to the profile of the dielectric grating that is at a maximum at the point of steepest gradient. Hence, polarization charge is induced at these points on the wax-air boundary, which in turn induces charge at points on the metal surface below [approximately at $k_{g} x=\frac{1}{2} \pi(2 p+1)$, where $p$ is an integer]. In the $p$-polarized case, there is clearly a component of $\mathbf{E}$ normal to the metal-wax interface. However, since it is a plane wave, one may initially think that all the charges induced should be in phase, yet a surface charge density oscillation at the metal boundary is induced. This is because of the difference in path length experienced by a photon striking the top of the highindex wax groove compared with one impinging near the trough. Hence we find that the charge centers are located symmetrically along the metal boundary with respect to the grating profile (at $k_{g} x=p \pi$ ), immediately below the peaks and troughs of the dielectric grating.

Of course at $\varphi=90^{\circ}$, and on excitation of the SPP, there will be two surface waves propagating in opposite directions along the metal surface. These two counterpropagating eigenmodes may be coupled together via two successive $k_{g}$ scattering events, setting up a standing wave in the $x$ direction, where the period of the standing wave is the same as the grating pitch $\left(\lambda_{\mathrm{SPP}}=\lambda_{g}\right)$. The standing wave associated with the $p$-coupled mode will have a different charge distribution on the surface of the metal from the charge distribution associated with the $s$-coupled mode, and hence two quite distinct field profiles will result. One field profile has maxima in $E_{y}$ below the peaks and troughs of the grating surface (coupled to via $p$-polarized incident radiation), the other has its extrema underneath the points of steepest gradient (coupled to via $s$-polarized radiation). These correspond to a low- and a high-energy mode, respectively, and hence an energy gap is seen to open up at $\varphi=90^{\circ}$.

The situation described above is further complicated since at the polar angle of incidence studied in this work ( $\theta$ $=80^{\circ}$ ), there may be coupling to the $s$-coupled SPP at two different energies (Fig. 4), both of which exist at lower energies than that needed to coupled to the $p$ mode. Therefore, it is interesting to theoretically model the dispersion of the SPP mode as a function of frequency and polar angle of incidence (Fig. 7). The boundary between the areas of low and high reflectivity, which forms an arc in the top left corner of Fig. 7, is associated with the \pm 2 Rayleigh anomaly, and the dark band that extends from the bottom left corner of both the graphs corresponds to the excitation of the \pm 2 SPP. This figure illustrates that one may only couple $s$-polarized radiation to a SPP mode at two different energies when $\sin \theta=k_{z} / k_{0}>0.9$ (approximately). Notice also that an 

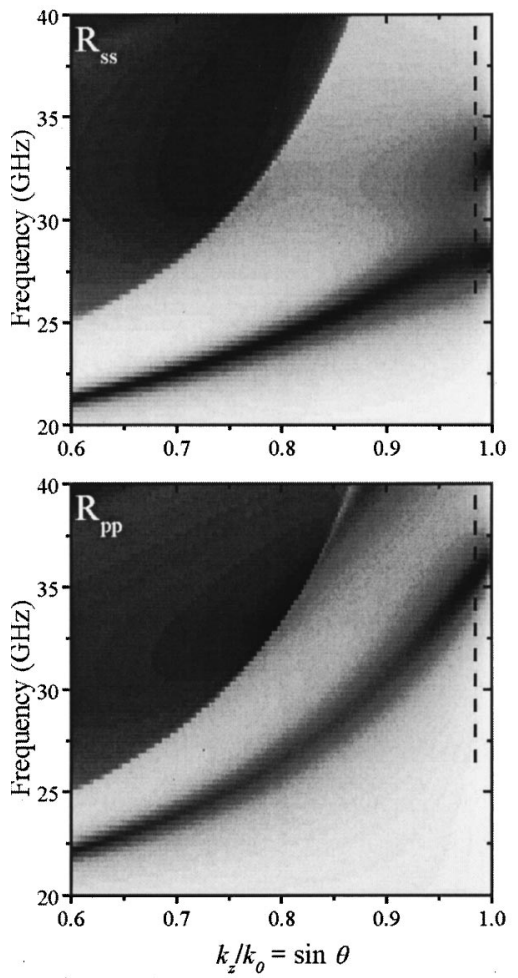

$0.9-1.0$

$0.8-0.9$

$0.7-0.8$

$0.6-0.7$

$0.5-0.6$

$0.4-0.5$

$0.3-0.4$

$0.2-0.3$

$0.1-0.2$

$0.0-0.1$

FIG. 7. Theoretical prediction of the $R_{s s}$ and $R_{p p}$ response of the sample illustrating the dispersion of the SPP mode as a function of incident momentum $\left(k_{z} / k_{0}=\sin \theta\right)$ and frequency. The data have been calculated using the fitting parameters derived earlier in the work and the vertical black dashed lines represent the experimental scans undertaken.

equivalent "splitting" of the $p$-coupled mode at high polar angles does not occur. To explain this result, it is useful to refer back to the vector field illustrations of Fig. 6 .

First consider the $p$-coupled mode, where the standing wave in the $x$ direction has its charge centers on the metal surface beneath the peaks and troughs of the dielectric grating. For $\theta \neq 0^{\circ}$, there are two loops of $E_{x y}$ within each grating period where neighboring loops are in antiphase [Fig. 6(c)]. Now consider the $s$-polarized case. When $\sin \theta \approx 0.75$, there is only one coupling condition to the SPP and again two loops of $E_{x y}$ are seen. However, since the standing wave at the metal interface is out of phase by $\lambda_{g} / 4$ with respect to the grating profile, one loop exists primarily within the wax and the other samples the air. As grazing incidence is approached $\left(k_{z} \rightarrow k_{0}\right)$, one of the field loops becomes dominant at the expense of the other. Eventually one of the loops almost disappears, and now neighboring loops of $\mathbf{E}$ are in phase with each other and two quite distinct bands are observed. Band 2 corresponds to the mode whose loops of $E_{x y}$ are almost entirely contained within the wax of the grating, and by contrast the field loops associated with band 3 are concentrated within the air [Figs. 6(a) and 6(b), respectively]. Since a mode whose field loops are concentrated in the wax samples a higher effective refractive index than one whose fields may penetrate into the air, modes associated with band 2 exist at a lower energy than those of band 3 . This is also clear from the experimental scan shown in Fig. 5 , which corresponds to the black dashed line superimposed on Fig. 7. Also evident from these figures is that band 2
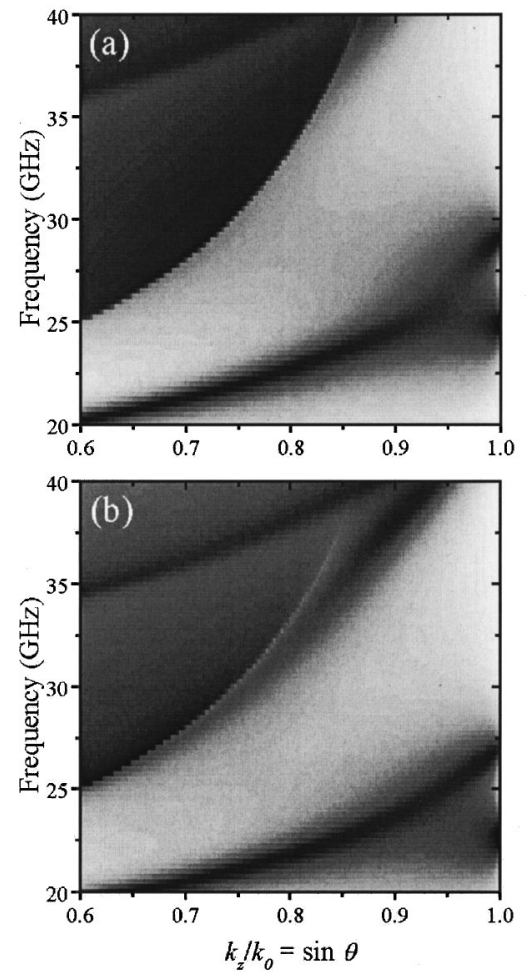

$0.9-1.0$

$0.8-0.9$

$0.7-0.8$

$0.6-0.7$

$0.5-0.6$

$0.4-0.5$

$0.3-0.4$

$0.2-0.3$

$0.1-0.2$

$0.0-0.1$

FIG. 8. Theoretical predictions of the $R_{s s}$ response of a similar sample to that used in this study but with thicker wax overlayers: (a) $t=2.10 \mathrm{~mm}$ and (b) $t=2.50 \mathrm{~mm}$.

appears to be the dominant one, and modes associated with it are more strongly coupled than modes associated with band 3 . However, one may change the relative coupling strengths of the two modes by varying the thickness of the corrugated overlayer. Figure 8 illustrates the dispersion of the modes with $\theta$ and frequency when (a) each of the two bands is equally well coupled $(t=2.10 \mathrm{~mm})$, and (b) band 3 becomes dominant $(t=2.50 \mathrm{~mm})$

It is important to note that, although $s$-polarized radiation is able to induce surface charge beneath the points of greatest gradient of the air-wax interface at $\varphi=90^{\circ}$ and hence couple strongly to modes 2 and 3 , there is no coupling in the $R_{p p}$ response and only weak coupling away from this orientation (Fig. 4). This is because incident $p$-polarized radiation is not able to induce the necessary surface charge when the grating is at this $\varphi$. In a similar manner, but for all grating orientations, it is impossible for s-polarized radiation to create surface charge beneath the peaks and troughs of the wax-air interface; hence mode 4 may only be excited with $p$-polarized radiation.

\section{CONCLUSIONS}

In this work near-grazing microwave radiation has been coupled via a dielectric grating to SPPs that propagate along a planar metal surface. The reflectivity of the sample is recorded as a function of azimuthal angle and incident wavelength in the microwave regime, using petroleum wax as the high-index dielectric. Good agreement between the predictions from a rigorous grating modeling theory and the experimental reflectivities has been obtained, and the fitting pro- 
cess allows verification of the profile of the grating and the dielectric constants of the wax at these frequencies.

It is shown that when the grating is oriented with its grooves parallel to the plane of incidence, coupling to a SPP via both incident $p$ - and $s$-polarized radiation is observed with an energy gap appearing between the two modes. Furthermore, at high polar angles of incidence, the $s$-coupled mode splits into two bands with different energies. The identity of each of the modes is confirmed as the excitation of the SPP, and an explanation is provided for their coupling con- ditions by modeling their resonant electric field profiles and their dispersion with frequency and in-plane momentum.

\section{ACKNOWLEDGMENTS}

The authors would like to acknowledge the financial support of the Defence Evaluation and Research Agency (DERA), Farnborough. This work was carried out as part of Technology Group 08 of the MoD Corporate Research Programme.
[1] R. W. Wood, Philos. Mag. 4, 396 (1902).

[2] R. A. Watts, J. R. Sambles, and J. B. Harris, Opt. Commun. 135, 189 (1997).

[3] A. Otto, Z. Phys. 216, 398 (1968).

[4] E. Kretschmann and H. Raether, Z. Naturforsch. A 23, 2135 (1968).

[5] R. H. Ritchie, E. T. Arakawa, J. J. Cowan, and R. N. Hamm, Phys. Rev. Lett. 21, 1530 (1968).

[6] H. Raether, Surface Plasmons on Smooth and Rough Surfaces and on Gratings (Springer, Berlin, 1988).

[7] G. N. Zhizhin, M. A. Moskalova, E. V. Shomina, and V. A. Yakovlev, Surface Polaritons: Electromagnetic Waves at Surfaces and Interfaces (North-Holland, Amsterdam, 1982).

[8] A. P. Hibbins, J. R. Sambles, and C. R. Lawrence, J. Appl. Phys. 86, 1791 (1999).

[9] R. A. Watts, T. W. Priest, and J. R. Sambles, Phys. Rev. Lett. 79, 3978 (1997).
[10] K. G. Müller, M. Veith, S. Mittler-Neher, and W. Knoll, J. Appl. Phys. 82, 4172 (1997).

[11] I. F. Salakhutdinov, V. A. Sychugov, and O. Parriaux, Quantum Electron. 28, 983 (1998).

[12] S. R. Seshadri, J. Opt. Soc. Am. A 16, 922 (1999).

[13] A. P. Hibbins, J. R. Sambles, and C. R. Lawrence, J. Appl. Phys. 87, 2677 (2000).

[14] R. C. McPhedran and M. D. Waterworth, Opt. Acta 19, 877 (1972).

[15] I. Pockrand, J. Phys. D 9, 2423 (1976).

[16] G. P. Bryan-Brown and J. R. Sambles, J. Mod. Opt. 37, 1227 (1990).

[17] T. W. Preist, N. P. K. Cotter, and J. R. Sambles, J. Opt. Soc. Am. A 12, 1740 (1995).

[18] J. Chandezon, M. T. Dupuis, G. Cornet, and D. Maystre, J. Opt. Soc. Am. 72, 839 (1982). 